\title{
RIGOROUS SOLUTION OF A DIFFERENTIAL EQUATION IN SOIL MECHANICS*
}

By R. GRAN OLSSON (Norwegian Institute of Technology, Trondheim)

In one of his earlier books $\mathrm{K}$. Terzaghi ${ }^{1}$ published a differential equation describing approximately the progress of consolidation in a sediment which is being deposited at a constant rate $q$ per unit of time. In connection with his solution of this equation Terzaghi pointed out that it does not satisfy the boundary conditions for time $t=0$. In the following paragraphs the writer presents a solution which satisfies the boundary conditions for any time, $t=0$ included.

Let $a_{v}\left[\mathrm{gm}^{-1} \mathrm{~cm}^{2}\right]$ be the coefficient of compressibility, $C$ a constant of integration, $c$ [sec] a time constant, $k\left[\mathrm{~cm} \mathrm{sec}^{-1}\right]$ the coefficient of permeability (Darcy's coefficient), $q\left[\mathrm{gm} \mathrm{cm}^{-2} \mathrm{sec}^{-1}\right]$ the quantity of sedimentation per unit of area and time, $t$ [sec] the time, $\gamma_{1}\left[\mathrm{gm} \mathrm{cm} \mathrm{cm}^{-3}\right]$ the unit weight, $\gamma_{w}\left[\mathrm{gm} \mathrm{cm}^{-3}\right]$ the unit weight of water $=1\left[\mathrm{gm} \mathrm{cm}^{-3}\right]$, $\gamma\left[\mathrm{gm} \mathrm{cm}{ }^{-3}\right]$ the submerged unit weight $\left(=\gamma_{1}-\gamma_{w}\right), \zeta$ the time function, $z=(c / t)^{1 / 2}$ a dimensionless independent variable, and $\phi(z)$ the error integral.

Using these symbols, which are identical with those used by Terzaghi, except that $\gamma_{w}$ represents the unit weight of water, the differential equation ${ }^{2}$ assumes the form

$$
\frac{d \zeta}{d t}+\frac{3}{t^{2}}\left(\frac{t}{2}+\frac{c}{3}\right) \zeta=\frac{c}{t^{2}}
$$

where $c=3 \gamma^{2} k / \gamma_{w} a q^{2}$ is a constant with the dimension time.

With

$$
z=(c / t)^{1 / 2}
$$

then

$$
t=c z^{-2}
$$

and

$$
d t=-2 c z^{-3} d z, \quad \frac{d \zeta}{d t}=-\frac{z^{3}}{2 c} \frac{d \zeta}{d z}
$$

substituting these values into Eq. (1), we obtain the equation

$$
\frac{d \zeta}{d z}=\left(\frac{3}{z}+2 z\right) \zeta-2 z
$$

This equation is solved in the usual way by first finding the complementary function from the homogenous equation

$$
\frac{d \zeta}{\zeta}=\left(\frac{3}{z}+2 z\right) d z
$$

Integrating we obtain

$$
\zeta(z)=C z^{3} \exp \left(z^{2}\right)
$$

where $C$ is an arbitrary constant.

*Received Oct. 27, 1948.

${ }^{1} \mathrm{~K}$. Terzaghi, Erdbaumechanik auf bodenphysikalischer Grundlage, Franz Deuticke, Leipzig and Vienna, 1924, p. 175.

${ }^{2}$ K. Terzaghi, op. cit., p. 175, Eq. (119). 
The general solution is given as

$$
\zeta(z)=C(z) z^{3} \exp \left(z^{2}\right)
$$

where $C(z)$ is an arbitrary function to be determined by (1a). Differentiating we get

$$
\frac{d \zeta}{d z}=\left[C^{\prime}(z) z^{3}+\left(3 z^{2}+2 z^{4}\right) C(z)\right] \exp \left(z^{2}\right)
$$

which inserted into (1a) yields,

$$
C^{\prime}(z)=-2 z^{-2} \exp \left(-z^{2}\right) .
$$

By integration we finally get

$$
C(z)=-2 \int \exp \left(-z^{2}\right) z^{-2} d z .
$$

To find the integral we integrate by parts:

$$
\int \exp \left(-z^{2}\right) z^{-2} d z=-\frac{\exp \left(-z^{2}\right)}{z}-2 \int \exp \left(-z^{2}\right) d z
$$

and thus

$$
C(z)=\frac{2}{z} \exp \left(-z^{2}\right)+4 \int \exp \left(-z^{2}\right) d z+C_{1},
$$

where $C_{1}$ is again an arbitrary constant, which is determined by the boundary condition for $t=0$.

It is convenient to introduce the error integral as a known (and tabulated) function ${ }^{3}$,

$$
\int \exp \left(-z^{2}\right) d z=\frac{1}{2} \pi^{1 / 2} \phi(z) .
$$

Thus we get for $C(z)$ the expression

$$
C(z)=\frac{2}{z} \exp \left(-z^{2}\right)+2 \pi^{1 / 2} \phi(z)+C_{1}
$$

and for the complete solution of the Eq. (1),

$$
\zeta(z)=2 z^{3} \exp \left(z^{2}\right)\left[z^{-1} \exp \left(-z^{2}\right)+\pi^{1 / 2} \phi(z)+\frac{1}{2} C_{1}\right] .
$$

The constant $C_{1}$ is determined from the boundary condition that for $t=0, \zeta=\zeta_{0}=1$. When $t \rightarrow 0, z \rightarrow \infty$, we resort to the asymptotic development of $\phi(z)$ in a semiconvergent series

$$
\phi(z)=1-\frac{\exp \left(-z^{2}\right)}{z}\left(1-\frac{1}{2 z^{2}}+\frac{1 \cdot 3}{\left(2 z^{2}\right)^{2}}-\frac{1 \cdot 3 \cdot 5}{\left(2 z^{2}\right)^{3}}+-\cdots\right)
$$

and get for $\zeta(z)$,

$$
\begin{aligned}
\zeta(z)=2 z^{3} \exp \left(z^{2}\right)\left[\frac{1}{z} \exp \left(-z^{2}\right)\right. & +\pi^{1 / 2}-\frac{1}{z} \exp \left(-z^{2}\right)\left(1-\frac{1}{2 z^{2}}\right. \\
& \left.\left.+\frac{1 \cdot 3}{\left(2 z^{2}\right)^{2}}-+\cdots\right)+\frac{1}{2} C_{1}\right] .
\end{aligned}
$$

${ }^{3}$ E. Jahnke and F. Emde, Tables of functions, Dover Publications, New York, 1945, p. 24. 
By putting $C_{1}=-2 \pi^{1 / 2}$ we get $\zeta=1$ when $z \rightarrow \infty$, since both constant terms and $z^{-1}$ $\exp \left(z^{2}\right)$ and $-z^{-1} \exp \left(z^{2}\right)$ cancel. We finally obtain

$$
\zeta_{z \rightarrow \infty}(z)=2 z^{3} \exp \left(z^{2}\right)\left(\frac{\exp \left(-z^{2}\right)}{2 z^{3}}-\frac{1 \cdot 3}{4 z^{5}} \exp \left(-z^{2}\right)+-\cdots\right)=1-\frac{3}{2 z^{2}}+-\cdots .
$$

This satisfies exactly the boundary condition $\zeta=1$ for $t=0$. The diagram shown in Fig. 1 gives $\zeta$ as a function of the non-dimensional "time" $t / c$. It is to be observed that

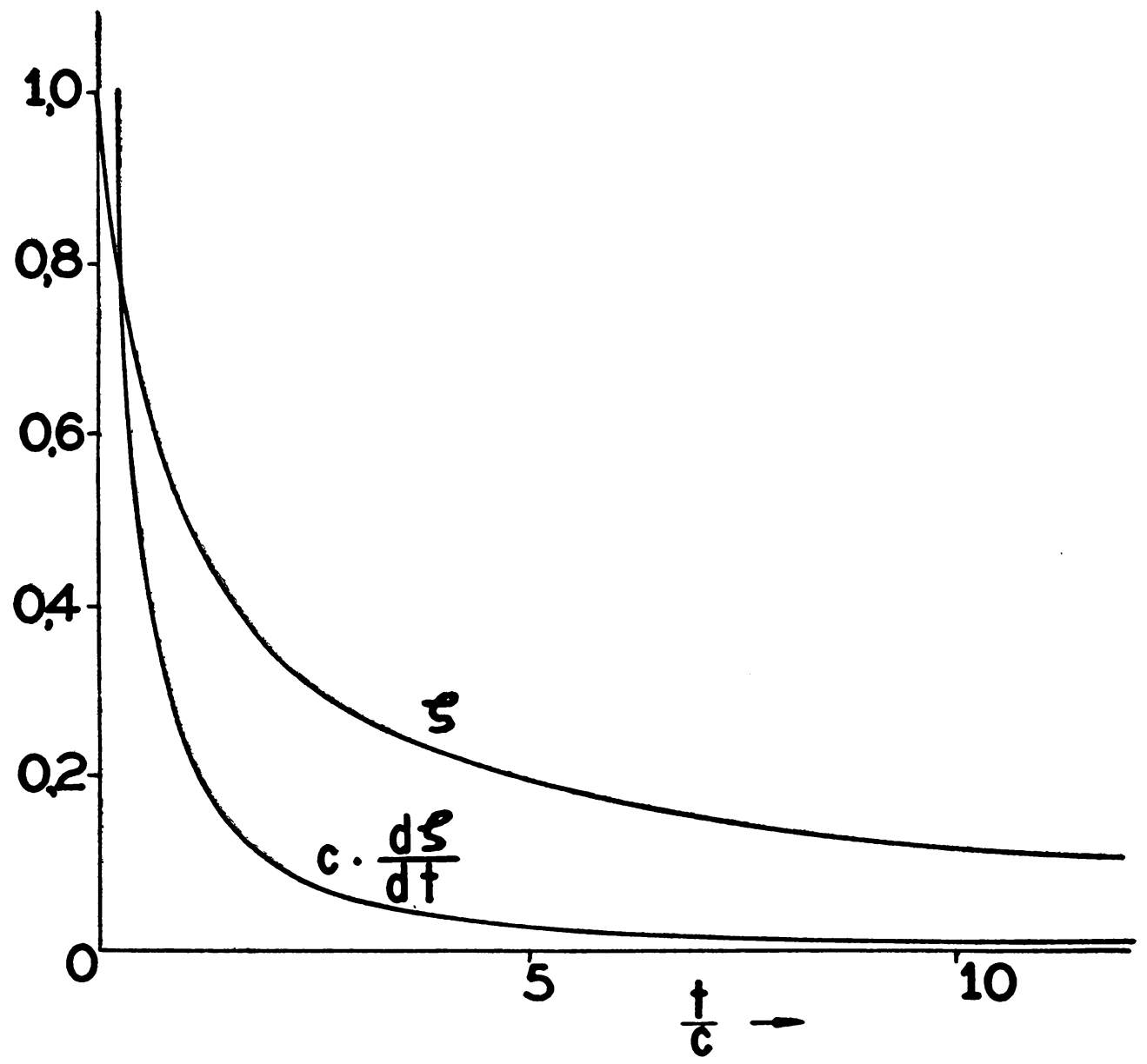

FIG. 1

for small values of $t / c$ we must use very accurately tabulated values of $\phi(z)$ because the function $\zeta$ appears as the difference between two nearly equal quantities. ${ }^{4}$ In general, the solution of the differential equation satisfying exactly the boundary condition $\zeta=1$ for $t=0$ will have the form

$$
\zeta(z)=2 z^{3} \exp \left(z^{2}\right)\left[z^{-1} \exp \left(-z^{2}\right)+\pi^{1 / 2} \phi(z)-\pi^{1 / 2}\right] .
$$

${ }^{4} \mathrm{~J}$. Burgess, On the definite integral $\left(2 / \pi^{1 / 2}\right) \int_{0}^{t} \exp \left(-t^{2}\right) d t$ with extended tables., Trans. Roy. Soc. Edingurgh 39 (Part II), (1898) 
This solution may be given a different form by introducing the derivative of the error function

$$
\phi^{\prime}(z)=\frac{2}{\pi^{1 / 2}} \exp \left(-z^{2}\right) \quad \text { or } \quad \exp \left(z^{2}\right)=\frac{2}{\pi^{1 / 2}}\left[\phi^{\prime}(z)\right]^{-1} .
$$

Thus we get

$$
\zeta(z)=4\left[\phi^{\prime}(z)\right]^{-1} z^{3}\left[\phi(z)+2 z^{-1} \phi^{\prime}(z)-1\right]
$$

as the most elegant form in which the solution may be written.

Besides $\zeta(z)$ the derivative $d \zeta / d t$ is also of interest. From the Eq. (1a) we get

$$
\frac{d \zeta}{d z}=4 z^{3}\left[\phi^{\prime}(z)\right]^{-1}\left[\phi(z)+2 z^{-1} \phi^{\prime}(z)-1\right]\left(3 z^{-1}+2 z\right)-2 z .
$$

Further we have

$$
\frac{d \zeta}{d t}=\frac{d \zeta}{d z} \frac{d z}{d t}=-\frac{z^{3}}{2 c} \frac{d \zeta}{d z}
$$

and finally

$$
\frac{d \zeta}{d t}=-\frac{2 z^{6}}{c}\left[\phi^{\prime}(z)\right]^{-1}\left[\phi(z)+2 z^{-1} \phi^{\prime}(z)-1\right]\left(3 z^{-1}+2 z\right)+\frac{z^{4}}{c} .
$$

The diagram in Fig. 1 gives $d \zeta / d t$ as a function of the independent variable $t / c$.

$A P P E N D I X$. When the sedimentation process is finished, the time factor increases according to the equation [1, p. 176, Eq. (122)]

$$
(1-\zeta) \exp \left(2 k t / a \gamma_{w} h_{1}^{2}\right)=\left(1-\zeta_{1}\right) \exp \left(2 k t_{1} / a \gamma_{w} h_{1}^{2}\right)
$$

or by introducing the "time constant"

$$
c=\frac{3 \gamma^{2} k}{\gamma_{w} a q^{2}}
$$

into the Eq. (1) we get

$$
(1-\zeta) \exp \left(2 c t / 3 t_{1}^{2}\right)=\left(1-\zeta_{1}\right) \exp \left(2 c / 3 t_{1}\right) .
$$

The time is so determined, that $t$ is equal to zero at the beginning of the sedimentation process and $t=t_{1}$ at its end. We obtain the value of $\zeta_{1}$ from the earlier solution of the differential equation, i.e.,

$$
\zeta_{1}=4 \phi^{\prime}\left(z_{1}\right)^{-1} z_{1}^{3}\left[\phi\left(z_{1}\right)+\frac{1}{2 z_{1}^{2}} \phi^{\prime}\left(z_{1}\right)-1\right],
$$

by introducing $z_{1}=\left(c^{7} / t_{1}\right)^{1 / 2}$ into the solution (3).

Equation (1b) determines a system of curves starting from a point $\left(c / t_{1}, \zeta_{1}\right)$ on the curve $\zeta$ and having the horizontal line $\zeta=1$ as an asymptote. Thus we get the system of curves represented in Fig. 2. It can easily be proved that there exists only one set of such curves according to the Eqs. (1) and (1b), so that the curves in Fig. 2 
are a general representation of the consolidation problem after finishing of the sedimentation process. In Fig. 2 the upper curves correspond to the greater values of $k / a$

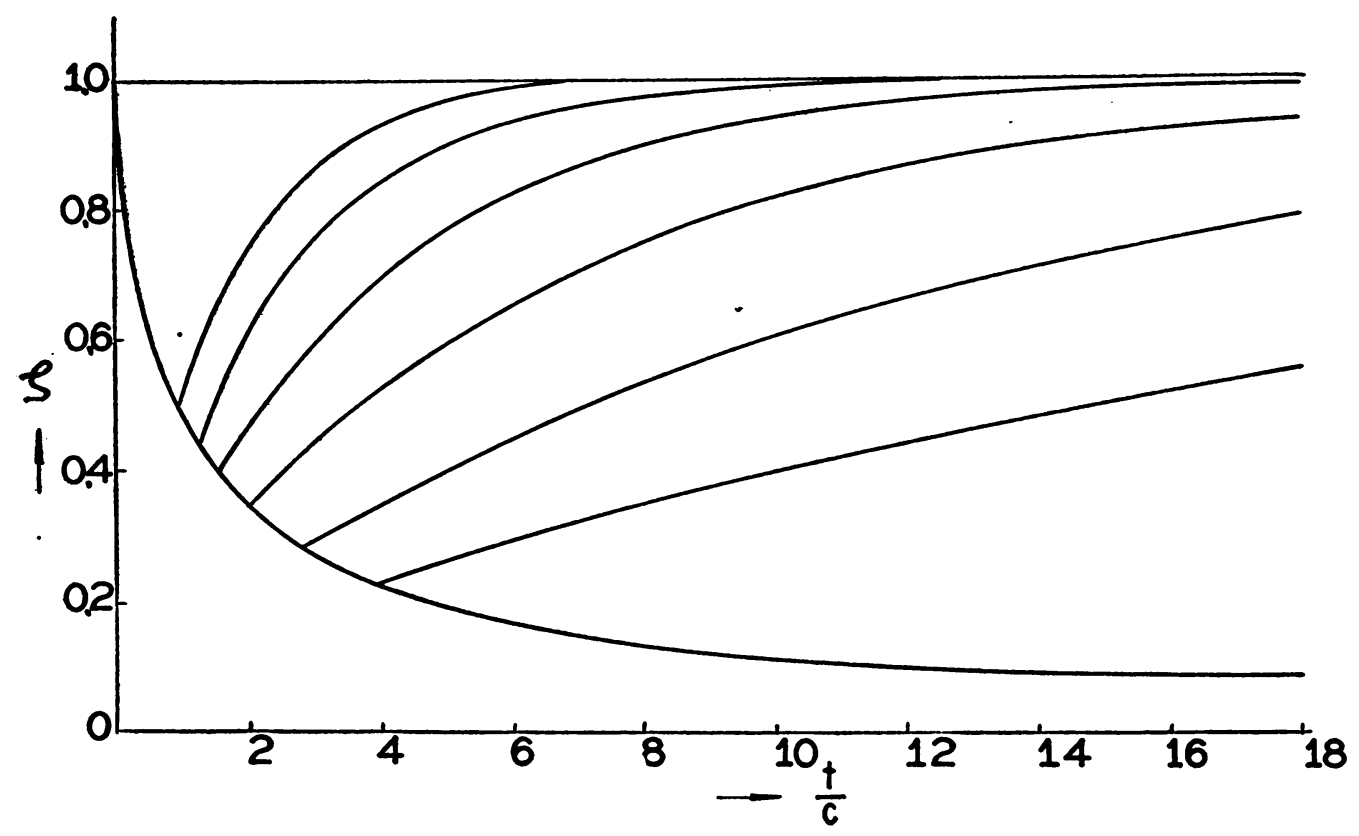

Fig. 2

as valid for sand and other permeable materials, whereas the lower curves correspond to clay and similar earth masses with smaller values of the coefficient of permeability.

\section{NOTE ON THE PROBLEM OF TWISTING OF A CIRCULAR RING SECTOR*}

BY ERIC REISSNER (Massachusetts Institute of Technology)

The problem of twisting of a circular ring sector is of some interest in connection with the calculation of stresses and deformations in close-coiled helical springs. To be considered is a ring-sector under the action of two equal and opposite forces $P$ along the axis through the center of the ring and perpendicular to the plane of the ring (Fig. 1). A formulation of the problem and an outline of results by O. Göhner for sectors of solid circular and rectangular cross section may be found on pp. 355-361 in Theory of elasticity by S. Timoshenko.

The purpose of the present note is to obtain explicit results for the twisting of ring sectors of hollow cross sections, with thin walls. Formulas will be obtained which have the same meaning for the present problem as R. Bredt's formulas have for the problem of St. Venant torsion of cylindrical rods.

The problem may be considered as one of the membrane theory of thin shells of

${ }^{*}$ Received Dec. 23, 1948. Work on this note was supported by the Office of Naval Research under Contract N5ori-07834. 